\title{
Effect of Nighttime Earplugs and Eye Masks on Sleep Quality in Intensive Care Unit Patients
}

\author{
Pureepat Arttawejkul ${ }^{1}$, Sirimon Reutrakul ${ }^{2}$, Dittapol Muntham ${ }^{3}$, Naricha Chirakalwasan ${ }^{4}$
}

\begin{abstract}
Purpose: Poor sleep quality in intensive care unit (ICU) can be associated with poor outcome. Excessive noise and lights in ICU are known to disrupt patients' sleep by causing arousals.

Study design: A prospective randomized controlled study.

Materials and methods: The patients admitted to the medical ICU were prospectively included and randomized to receive earplugs and eye masks or no intervention during their first 5 nights in ICU. Their arousal index and other sleep parameters were measured during the first night by polysomnography. Secondary outcomes including wrist actigraphy profiles and subjective sleep quality were recorded during all study nights. Results: Seventeen patients were enrolled. Eight patients were randomized to earplugs and eye masks group and nine patients were randomized to control group during their first 5 nights in the ICU. The use of earplugs and eye masks demonstrated the trend toward lower arousal index during the first night (21.15 (14.60) vs 42.10 (18.20) events per hour, $p=0.086)$ and increased activity index (activity count/hour) (16.12 (7.99) vs 10.84 (10.39) count/hour, $p=0.059$ ) compared to control group. Polysomnography and actigraphy did not demonstrate good agreement. Conclusion: The use of earplugs and eye masks has a trend toward reduction in arousal index and increased activity in patients admitted to ICU. Limited sample size most likely explained insignificant difference in outcomes. Wrist actigraphy did not accurately measure sleep parameters in ICU patients.

Trial registration: www.clinicaltrials.in.th, TCTR20170727003.

Keywords: Delirium, Earplugs, Eye masks, Intensive care unit, Light and noise control, Sleep quality.

Indian Journal of Critical Care Medicine (2020): 10.5005/jp-journals-10071-23321
\end{abstract}

\section{INTRODUCTION}

Sleep during critical illness is known to be poor ${ }^{1}$ secondary to several factors including pain, anxiety, medication side effect, ventilator dyssynchrony, and nurse's intervention. ${ }^{2}$ Noises and light disturbances are known to disrupt sleep in intensive care unit (ICU). ${ }^{3}$ The noise in the ICU was demonstrated to be in part responsible for sleep-wake abnormalities. ${ }^{1}$ Theoretically, poor sleep quality may reduce muscle endurance resulting in prolonged weaning. ${ }^{4}$ Delirium can also be observed with sleep deprivation in ICU associated with increased mortality and long-term cognitive function impairment. ${ }^{5}$ Furthermore, sleep deprivation may also reduce immune response and increase risk of nosocomial infection. ${ }^{6}$

Intervention to improve sleep quality in ICU includes medical and nonmedical interventions. ${ }^{7}$ However, sedation should be used cautiously due to potential prolongation of mechanical ventilation. ${ }^{8}$ Environmental modifications were demonstrated to improve perceived sleep quality and cognition. ${ }^{9}$ Ambient light and noise reduction were proven to improve sleep quality. ${ }^{10}$ However, these modalities may require complicated hospital environmental and work schedule modification.

Simple interventions including earplugs and eye masks have been shown to be beneficial in postoperative patients in surgical ICU. In this study, the intervention group was observed to have better sleep quality, required less hypnotics and analgesics, and less delirium. ${ }^{11}$ Currently, there have been no studies using these simple interventions in medical ICU. Given different patient characteristics and environmental factors between surgical and medical ICU, we aimed to study the effect of earplugs and eye masks on sleep quality and related ICU outcomes in medical ICU setting.

\begin{abstract}
1,4 Department of Medicine, Division of Pulmonary and Critical Care Medicine, Faculty of Medicine, Chulalongkorn University, Bangkok, Thailand; Excellence Center for Sleep Disorders, King Chulalongkorn Memorial Hospital, Thai Red Cross Society, Bangkok, Thailand

${ }^{2}$ Department of Medicine, Division of Endocrinology, Diabetes and Metabolism, University of Illinois at Chicago, Chicago, Illinois, USA

${ }^{3}$ Excellence Center for Sleep Disorders, King Chulalongkorn Memorial Hospital, Thai Red Cross Society, Bangkok, Thailand; Section for Mathematics, Faculty of Science and Technology, Rajamangala University of Technology Suvarnabhumi, Phra Nakhon Si Ayutthaya, Thailand
\end{abstract}

Corresponding Author: Naricha Chirakalwasan, Department of Medicine, Division of Pulmonary and Critical Care Medicine, Faculty of Medicine, Chulalongkorn University, Bangkok, Thailand; Excellence Center for Sleep Disorders, King Chulalongkorn Memorial Hospital, Thai Red Cross Society, Bangkok, Thailand, Phone: +66-2-649-4037, email: narichac@hotmail.com

How to cite this article: Arttawejkul P, Reutrakul S, Muntham D, Chirakalwasan N. Effect of Nighttime Earplugs and Eye Masks on Sleep Quality in Intensive Care Unit Patients. Indian J Crit Care Med 2020;24(1):6-10.

Source of support: This research has been supported by the Ratchadaphiseksomphot Endowment Fund of Chulalongkorn University

Conflict of interest: None

\section{Materials and Methods}

The study was a randomized controlled trial. The patients admitted to medical ICU (single unit, total of 16 beds) and expected to remain 
in ICU for at least 24 hours who were at least 18 years of age, able to understand Thai, and can communicate with investigator, and Glasgow coma score $\geq 13$, Richmond agitation-sedation score was -1 to +1 , and did not require medication or intervention to facilitate sleep were enrolled in our study within 24 hours of their ICU admission. Exclusion criteria were bilateral deafness, bilateral blindness, severe encephalopathy, severe dementia, hepatic encephalopathy, uremic encephalopathy, encephalitis, increased intracranial pressure, metabolic derangements, severe hemodynamic instability, high vasopressor requirement (dopamine $>15 \mu \mathrm{g} / \mathrm{kg} /$ minute, epinephrine $>0.1 \mu \mathrm{g} / \mathrm{kg} /$ minute, and norepinephrine $>0.1 \mu \mathrm{g} / \mathrm{kg} /$ minute for at least 1 hour), and severe respiratory failure $\left(\mathrm{PaO}_{2} / \mathrm{FiO}_{2}<100\right)$. Our medical ICU work environment includes nurse to patient ratio of 1:1 (8-hour shift) and standard hospital environment including 24-hour hospital lightings and no sound level control. The patients were randomized by stratified block randomization to receive earplugs and eye masks or to be in control group.

\section{Intervention}

In the study group, the patients were given earplugs (noise reduction rating of $32 \mathrm{~dB}$ ) and cloth eye masks by physician or nurse during sleep at nighttime according to their habitual home bedtime but not after 22:00 hours. Earplugs and eye masks were removed at 07:00 hours on the following morning. Earplugs and eye masks were allowed to be removed for no longer than 10 minutes for communication if needed. If the patients did not wish to continue with the use of these interventions, they were allowed to inform the nurse (verbal communication if not intubated and bell ringing if intubated) and the inventions can be stopped upon patient request. The patients were informed to use earplugs and eye masks every night during the ICU stay. We also obtained therapeutic intervention scoring system (TISS-28 score) in order to monitor the nurse's activity involving in medical care for each patient. ${ }^{12}$

\section{Polysomnography}

During the first night of the admission to the ICU, both study group and control group underwent type I polysomnography conducted in medical ICU. The polysomnography was conducted using standard EEG including frontal leads (F1, F2), central leads (C3, C4), occipital leads $(\mathrm{O} 1, \mathrm{O} 2)$, and reference leads at mastoids (M1, M2), electromyography, and electrooculography methodologies. The polysomnography was conducted at the patient's habitual home bedtime but not after 22:00 hours and the study was concluded at 07:00 hours in the following morning. Sleep stages were scored by board certified sleep medicine specialist blinded to the randomization using 30 -second epoch window according to the standard criteria from the American Academy of Sleep Medicine (AASM) Manual for the Scoring of Sleep and Associated Events, 2016. ${ }^{13}$

\section{Actigraphy}

Actiwatch $^{\circledast} 2$ (Respironics) was worn on patient's nondominant wrist throughout study duration. These monitors use highly sensitive omnidirectional accelerometers to count the number of wrist movements in 30-second epochs. This device also has light sensor, which records the light intensity and report this data in lux. The software scores each 30-second epoch as sleep or wake based on a threshold of activity counts that is estimated using activity within the epoch being scored as well as the epochs 2 minutes before and after that epoch. Total sleep time was defined as the amount of actual sleep obtained at night. Sleep efficiency was the percentage of time in bed spent sleeping. Sleep latency was defined as the time period from bedtime to the first epoch of sleep. Wake after sleep onset was defined as the time spent awake after the patient was asleep and before the patient was awake. All parameters were calculated using Actiware 6.0 software, supplied by the manufacturer. The investigator who scored the Actiwatch's data was blinded to the group allocation and the polysomnography data. The patients wore actigraphy during the entire duration in the study while they were in the ICU.

\section{Questionnaires}

Sleep history and sleep quality were obtained using Pittsburgh sleep quality index questionnaire (PSQI) ${ }^{14}$ as baseline at the enrollment period. In order to accurately monitor sleep quality in the ICU, both Richard-Campbell sleep questionnaire ${ }^{15}$ (RCSQ) and Verran/Snyder-Halpern sleep scale ${ }^{16}$ were utilized the following morning after the polysomnography and every morning for 5 days during the ICU stay or terminated earlier if the patient was discharged from the ICU before 5 days. In order to assess for the presence of delirium, CAM-ICU was utilized during the study for 5 days during the ICU stay or terminated earlier if the patient was discharged from the ICU before 5 days.

\section{Primary Outcome of the Study}

We aimed to determine the difference in arousal index compared between the group using earplugs and eye masks as primary outcome. We also compared other polysomnographic parameters, actigraphy parameters, sleep quality, the prevalence of delirium, the sedation requirement, duration of mechanical ventilation, rate of nosocomial infection, and duration of ICU stay.

\section{Statistical Analysis}

The study was analyzed as intention to treat analysis. Sample size was calculated from arousal index using mean difference between two independent samples derived from Huang et al. paper. ${ }^{17} \mathrm{We}$ used $\alpha=0.05$, and $\beta=0.20$, and calculated to have 6 patients in each arm (total of 12 patients). We also added $30 \%$ for dropout rate and another $30 \%$ for potential uninterpretable data. Eventually, a total of 20 patients were planned. Quantitative variables were compared between two groups using $t$ test or Mann-Whitney $U$ test and linear regression analysis. Chi-squared or Fisher's exact test was used to compare proportion between groups. Correlation analysis between polysomnography and Actiwatch parameters was analyzed using Lin's concordance correlation coefficient (0.21-0.40 = fair, $0.41-0.60=$ moderate, $0.61-0.80=$ substantial, and $0.81-1.00=$ almost perfect). STATA v12.1 software was utilized. Descriptive analysis was used for analysis. This study was approved by the Ethics Committee. The study was registered at www.clinicaltrials. in.th (\#TCTR20170727003).

\section{Results}

During the period of June 2017 to May 2018, a total of 20 subjects were enrolled in the study. Ten patients were randomized to control group and 10 patients were randomized to intervention group (earplugs and eye masks). Two patients in the intervention group were excluded (poor polysomnographic quality and ICU discharge prior to the conduct of polysomnography). One patient in control group was excluded due to uninterpretable polysomnography data. A total of 17 patients were analyzed. Baseline clinical characteristics were similar between two groups. Most of the patients had poor 
sleep quality as baseline according to PSQI. Clinical characteristic and information upon admission are listed in Table 1. The most common primary diagnosis for ICU admission was pneumonia.

Arousal index during the first night of the study demonstrated a trend toward lower value in the intervention group compared to control group after adjustment for overnight nursing interventions (TISS-28) $(p=0.086)$ (Fig. 1).

Other polysomnographic parameters including total sleep time, sleep efficiency, wake after sleep onset, sleep latency, \% rapid eye movement (REM) sleep, and \% N3 sleep were similar between two groups (Table 2).

Subjective sleep quality according to RCSQ score did not demonstrate the difference between the intervention group and the control group ( $p=0.236)$. The prevalence of delirium, the use of sedation, duration of ICU stay, and duration of mechanical ventilation were not different between two groups (Table 3).

Actiwatch data demonstrates a trend toward more activity in the intervention group (Table 4).

Table 1: Clinical characteristic and information upon admission of control and intervention groups

\begin{tabular}{lll}
\hline Clinical characteristics & $\begin{array}{l}\text { Control } \\
(n=9)\end{array}$ & $\begin{array}{l}\text { Intervention } \\
(n=8)\end{array}$ \\
\hline Sex & & \\
$\quad$ Male, $n(\%)$ & $5(56 \%)$ & $6(75 \%)$ \\
$\quad$ Female, $n$ (\%) & $4(44 \%)$ & $2(25 \%)$ \\
Age (years) & $76(32)^{*}$ & $67(25)^{*}$ \\
BMI & $21.23(5.42)^{*}$ & $21.35(4.74)^{*}$ \\
Sedation use, $n$ (\%) & $1(11 \%)$ & $1(13 \%)$ \\
Smoking (pack-year) & $0(10)^{*}$ & $14(20)^{*}$ \\
Alcohol use, $n$ (\%) & $2(22 \%)$ & $1(13 \%)$ \\
Primary diagnosis, pneumonia (\%) & $4(44.4 \%)$ & $3(37.5 \%)$ \\
Direct admission to ICU, $n$ (\%) & $5(56 \%)$ & $5(63 \%)$ \\
APACHE II & $14(1)^{*}$ & $15(7)^{*}$ \\
SOFA & $2(2)^{*}$ & $4(6)^{*}$ \\
Pittsburgh sleep quality index & $7(2)^{*}$ & $6(6)^{*}$ \\
Nights in the study & $2(1)^{*}$ & $4(2)^{*}$ \\
TISS-28 score during the first night & $19(3)^{*}$ & $24(7)^{*}$ \\
Light exposure & $27.77(16.93)^{*}$ & $52.68(48.44)^{*}$ \\
\hline
\end{tabular}

*Data was demonstrated in median (IQR)

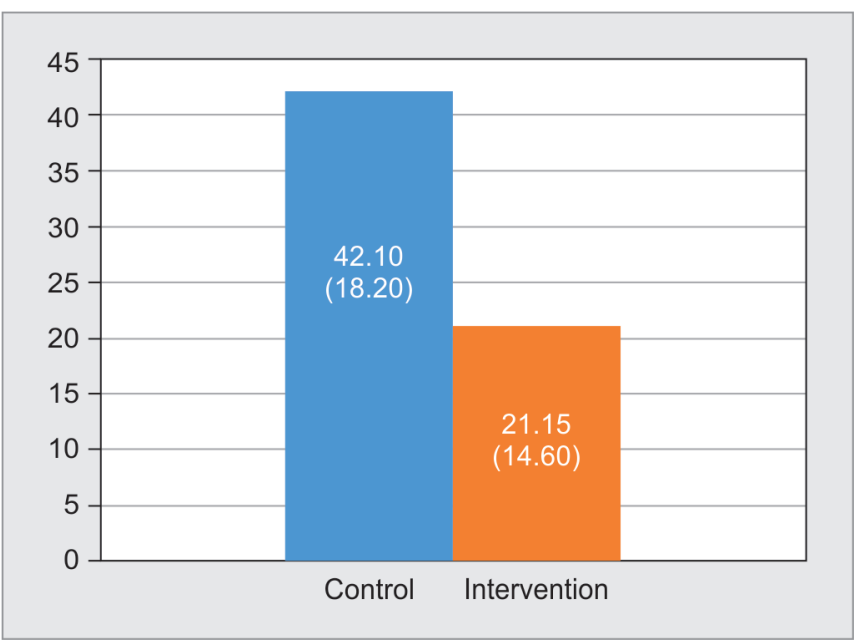

Fig. 1: Arousal index during the first night of the study
Overall correlation between polysomnography and actigraphy was poor to fair. Polysomnography and wrist actigraphy data showed fair agreement on sleep latency (correlation coefficient $=$ 0.389 ) and sleep efficiency (correlation coefficient $=0.223$ ) but poor agreement on total sleep time (correlation coefficient $=0.188$ ) and wake after sleep onset (correlation coefficient $=0.031$ ). Wake after sleep onset appeared to have the poorest correlation with polysomnography (Table 5).

\section{Discussion}

Our study conducted as a randomized controlled trial on the efficiency of the use of earplugs and eye masks and sleep quality

Table 2: Polysomnography data of control and intervention groups

\begin{tabular}{llll}
\hline PSG parameter & Control $(n=9)$ & Intervention $(n=8)$ & p value \\
\hline Total sleep time & $333(112)^{*}$ & $319(174)^{*}$ & 0.452 \\
Sleep efficiency & $77.10(9.70)^{*}$ & $65.2(32.85)^{*}$ & 0.891 \\
$\begin{array}{l}\text { Wake after sleep } \\
\text { onset }\end{array}$ & $114(107)^{*}$ & $190(175.5)^{*}$ & 0.698 \\
$\begin{array}{l}\text { Sleep latency } \\
\text { REM sleep }\end{array}$ & $14(21)^{*}$ & $0(22)^{*}$ & 0.368 \\
$\begin{array}{l}\text { percentage } \\
\begin{array}{l}\text { Slow wave sleep } \\
\text { percentage }\end{array}\end{array}$ & $1.3(22.1)^{*}$ & $3(31.6)^{*}$ & 0.608 \\
\end{tabular}

*Data was demonstrated in median (IQR) which was adjusted for TISS-28

${ }^{\S} p$ value was adjusted for overnight nursing interventions (TISS-28)

Table 3: Clinical outcomes of control and intervention groups

\begin{tabular}{llll}
\hline Clinical parameter & Control $(n=9)$ & Intervention $(n=8)$ & $p$ value \\
\hline RCSQ score & $56.4(5.17)^{\S}$ & $58.5(5.26)^{\S}$ & 0.236 \\
Delirium & $1(11.1 \%)$ & $1(12.5 \%)$ & 1.000 \\
$\begin{array}{l}\text { Sedation use } \\
\text { ICU duration } \\
\text { (hours) }\end{array}$ & $0(0 \%)$ & $1(12.5 \%)$ & 0.471 \\
$\begin{array}{l}\text { Mechanical } \\
\text { ventilation (hours) }\end{array}$ & $68(20)^{*}$ & $96(66.5)^{*}$ & 0.572 \\
$\begin{array}{l}\text { Nosocomial } \\
\text { infection }\end{array}$ & $0(0 \%)$ & $72.5(47.5)^{*}$ & 0.925 \\
$\begin{array}{l}\text { *Data was demonstrated in median (IQR) } \\
\text { \$Data was demonstrated in mean (SD) }\end{array}$ & $0(0 \%)$ & - \\
\hline
\end{tabular}

Table 4: Activity data from Actiwatch of control and intervention groups

\begin{tabular}{llll}
\hline $\begin{array}{l}\text { Actiwatch } \\
\text { parameter }\end{array}$ & Control $(n=9)$ & Intervention $(n=8)$ & $p$ value \\
\hline Activity count & $2529.25(887.23)^{*}$ & $5872.95(2611.53)^{*}$ & 0.093 \\
Activity index** $^{*}$ & $10.84(10.39)^{*}$ & $16.12(7.99)^{*}$ & 0.059
\end{tabular}

*Data was demonstrated in median (IQR)

${ }^{* *}$ Activity index = activity count per hour

Table 5: Correlation of actigraphy parameters and polysomnography parameters

\begin{tabular}{lll}
\hline $\begin{array}{l}\text { Polysomnography } \\
\text { parameter }\end{array}$ & $\begin{array}{l}\text { Actigraphy } \\
\text { parameter }\end{array}$ & $\begin{array}{l}\text { Correlation } \\
\text { coefficient }\end{array}$ \\
\hline Total sleep time & Actual sleep time & 0.188 \\
Sleep efficiency & Sleep efficiency & 0.223 \\
Wake after sleep onset & Wake after sleep onset & 0.031 \\
Sleep latency & Sleep latency & 0.389 \\
\hline
\end{tabular}


in real medical ICU using polysomnography. The previous studies measured sleep quality only by subjective questionnaire. ${ }^{18-20}$ Three previous studies using polysomnography were conducted in healthy subjects in simulated ICU environment. ${ }^{17,21,22}$ One study was conducted with earplugs ${ }^{21}$ and two studies were conducted with earplugs and eye masks. ${ }^{17,22}$ All previous studies using objective polysomnography to measure the efficacy of earplugs and eye masks on sleep quality in ICU were done only in simulated ICU environment and may not represent the full auditory and visual experience of the ICU.

Our study demonstrated a trend toward lower arousal index 21.15 (14.60) in intervention group compared to 42.10 (18.20) in control group ( $p=0.086$ ). This finding supports the benefit of noise and light reduction in promoting continuity of sleep in ICU setting. Similar to our finding, Hu et al. studied 14 healthy subjects exposed to recorded ICU noise and light and demonstrated that the use of earplugs and eye masks was associated with reduction in arousal index. ${ }^{22}$ Reduction in arousal index may clinically benefit since prior studies demonstrated detrimental effect of arousal. ${ }^{23-25}$ Prior study conducted in healthy volunteers demonstrated that arousal index is a single strongest polysomnographic predictor of daytime sympathetic discharge which could pose risk of increased blood pressure. ${ }^{23}$ In studies conducted in obstructive sleep apnea population, arousal index was demonstrated to be marker of carotid artery atherosclerosis, ${ }^{24}$ risk of acute coronary syndrome, transient ischemic attack, and stroke or death. ${ }^{25}$

Furthermore, our study also demonstrated a trend toward increase in activity with the use of earplugs and eye masks compared to control group ( $p=0.059)$. Winkelman et al. studied ventilated subjects in medical and surgical ICU using actigraphy and demonstrated that activity appeared to be associated with a decrease in IL-6 level. ${ }^{26}$ The same group also conducted another study in medical ICU and step-down unit on ventilated subjects participated in an early mobility program. ${ }^{27}$ The study demonstrated that lower activity count from actigraphy has the negative impact with potential toward alteration in inflammatory profiles. ${ }^{27}$ Clinical importance of increased activity of ICU patients was also previously demonstrated. Early immobilization was shown to reduce ICU and hospital lengths of $\mathrm{stay}_{1}{ }^{28}$ increase ventilator free days, ${ }^{29}$ reduce ICU-acquired weakness, ${ }^{29,30}$ increase proportion to return to independent function at discharge, ${ }^{31}$ reduce proportion of time in ICU with delirium, ${ }^{31}$ and improve glycemic control. ${ }^{30}$

Insignificant difference in the outcomes can be explained by several reasons. First, the limited sample size; the calculated sample size was from the study of healthy volunteers wearing earplugs and eye masks in simulated ICU setting with large difference between the intervention group and control group. Statistically, this large difference resulted in small calculated sample. This calculated sample size could have been inadequate since the difference between intervention group and control group in real ICU setting with more disturbing environment could have been less. In fact, larger sample size may be needed to detect the difference in expected outcomes. Second, inhomogeneous baseline characteristics between two groups could be potential explanation. Despite the randomization, the data indicated a trend toward less clinical severity (lower sequential organ failure assessment (SOFA) score) and lower light exposure during sleep observed in the control group compared to the intervention group. These differences may have promoted overall better sleep quality in the control group compared to the intervention group and masked the expected difference in the outcome. Finally, there are potential occult factors, which may have affected the outcome including various medication, procedures, or mode of medical ventilation that were not controlled in our study.

Although wrist actigraphy has long been used to measure sleep in ambulatory patients with great reliability, poor correlation between polysomnography and actigraphy in sleep measurement was in agreement with current evidence in the literature. Actigraphy was observed to be valid and reliable for detecting sleep in healthy adult populations but less reliable for detecting sleep in disturbed setting. ${ }^{32}$ Actigraphy may incorrectly score wakefulness as sleeping in ICU patients as they were awake but immobile due to restraints, sedation, or severe illness. ${ }^{33}$ Recent systematic review of 13 eligible studies also concluded that since actigraphy only measures gross motor activity, it is not recommended to be used to measure sleep in ICU. ${ }^{34}$ Algorithm of software used to analyze this data in actigraphy may have to be corrected for other variables before this device can be used accurately in ICU setting.

Our study suggests that polysomnography is still a gold standard for sleep measurement in ICU setting. Knauert et al. conducted polysomnography in 29 patients in medical ICU and demonstrated sufficient data to determine sleep stage, sleep efficiency, and arousal indices in their studies with 93\% interpretable quality. ${ }^{35}$ Similarly, our study demonstrated interpretable quality of polysomnography data to be $94 \%$.

\section{Conclusion}

Earplugs and eye masks insignificantly reduced arousal index and increased activity in patients admitted to ICU. Limited sample size most likely explained insignificant difference in outcomes in our study and larger sample size is needed. Wrist actigraphy did not accurately measure sleep parameters in ICU patients. To date, polysomnography is still a valid and reliable method to measure sleep parameters in ICU setting.

\section{Acknowledgment}

The study was conducted at King Chulalongkorn Memorial Hospital, Thai Red Cross Society, Bangkok, Thailand. This research was supported by the Ratchadaphiseksomphot Endowment Fund of Chulalongkorn University.

\section{References}

1. Freedman NS, Gazendam J, Levan L, Pack Al, Schwab RJ. Abnormal sleep/wake cycles and the effect of environmental noise on sleep disruption in the intensive care unit. Am J Respir Crit Care Med 2001;163(2):451-457. DOI: 10.1164/ajrccm.163.2.9912128.

2. Bihari S, Doug McEvoy R, Matheson E, Kim S, Woodman RJ, Bersten $A D$. Factors affecting sleep quality of patients in intensive care unit. J Clin Sleep Med 2012;8(3):301-307. DOI: 10.5664/jcsm.1920.

3. Gabor JY, Cooper AB, Crombach SA, Lee B, Kadikar N, Bettger HE, et al. Contribution of the intensive care unit environment to sleep disruption in mechanically ventilated patients and healthy subjects. Am J Respir Crit Care Med 2003;167(5):708-715. DOI: 10.1164/ rccm.2201090.

4. Chen HI, Tang YR. Sleep loss impairs inspiratory muscle endurance. Am Rev Respir Dis 1989;140(4):907-909. DOI: 10.1164/ajrccm/140.4.907.

5. Weinhouse GL, Schwab RJ, Watson PL, Patil N, Vaccaro B, Pandharipande $P$, et al. Bench-to-bedside review: delirium in ICU patients - importance of sleep deprivation. Crit Care 2009;13(6):234. DOI: $10.1186 /$ cc8131.

6. Spiegel K, Sheridan JF, Van Cauter E. Effect of sleep deprivation on response to immunization. JAMA 2002;288(12):1471-1472. DOI: 10.1001/jama.288.12.1469. 
7. Kamdar BB, King LM, Collop NA, Sakamuri S, Colantuoni E, Neufeld $\mathrm{KJ}$, et al. The effect of a quality improvement intervention on perceived sleep quality and cognition in a medical ICU. Crit Care Med 2013;41(3):800-809. DOI: 10.1097/CCM.0b013e3182746442.

8. Kollef MH, Levy NT, Ahrens TS, Schaiff R, Prentice D, Sherman G. The use of continuous i.v. sedation is associated with prolongation of mechanical ventilation. Chest 1998;114(2):541-548. DOI: 10.1378/ chest.114.2.541.

9. Hu RF, Jiang XY, Chen J, Zeng Z, Chen XY, Li Y, et al. Nonpharmacological interventions for sleep promotion in the intensive care unit. Cochrane Database Syst Rev 2015;10:CD008808. DOI: 10.1002/14651858.CD008808.pub2.

10. DuBose JR, Hadi K. Improving inpatient environments to support patient sleep. Int J Qual Health Care 2016;28(5):540-553. DOI: 10.1093/ intqhe/mzw079.

11. Le Guen M, Nicolas-Robin A, Lebard C, Arnulf I, Langeron O. Earplugs and eye masks vs routine care prevent sleep impairment in post-anaesthesia care unit: a randomized study. $\mathrm{Br} J$ Anaesth 2014;112(1):89-95. DOI: 10.1093/bja/aet304.

12. Miranda DR, de RijkA, Schaufeli W. Simplified therapeutic intervention scoring system: the TISS-28 items-results from a multicenter study. Crit Care Med 1996;24(1):64-73. DOI: 10.1097/00003246-19960100000012.

13. Berry BB, Brooks R, Gamaldo CE, Harding SM, Lloyd RM, Marcus CL, et al. For the American Academy of Sleep Medicine. In: The ASSM Manual for the Scoring of Sleep and Associated Events: Rules, Terminology and Technical Specifications. Version 2.3. Darien, Illinois: American Academy of Sleep Medicine; 2016.

14. Sitasuwan $T$, Bussaratid $S$, Ruttanaumpawan $P$, Chotinaiwattarakul W. Reliability and validity of the Thai version of the Pittsburgh Sleep Quality Index. J Med Assoc Thai 2014;97(Suppl 3):S57-S67.

15. Richards KC, O'Sullivan PS, Phillips RL. Measurement of sleep in critically ill patients. J Nurs Meas 2000;8(2):131-144. DOI: 10.1891/10613749.8.2.131.

16. Snyder-Halpern R, Verran JA. Instrumentation to describe subjective sleep characteristics in healthy subjects. Res Nurs Health 1987;10(3):155-163. DOI: 10.1002/nur.4770100307.

17. Huang HW, Zheng BL, Jiang L, Lin ZT, Zhang GB, Shen $L$, et al. Effect of oral melatonin and wearing earplugs and eye masks on nocturnal sleep in healthy subjects in a simulated intensive care unit environment: which might be a more promising strategy for ICU sleep deprivation? Crit Care 2015;19:124. DOI: 10.1186/s13054-0150842-8.

18. Van Rompaey B, Elseviers MM, Van Drom W, Fromont V, Jorens PG. The effect of earplugs during the night on the onset of delirium and sleep perception: a randomized controlled trial in intensive care patients. Crit Care 2012;16(3):R73. DOI: 10.1186/cc11330.

19. Daneshmandi M, Neiseh F, SadeghiShermeh M, Ebadi A. Effect of eye mask on sleep quality in patients with acute coronary syndrome. J Caring Sci 2012;1(3):135-143. DOI: 10.5681/jcs.2012.020.

20. Babaii A, Adib-Hajbaghery M, Hajibagheri A. Effect of using eye mask on sleep quality in cardiac patients: a randomized controlled trial. Nurs Midwifery Stud 2015;4(4):e28332. DOI: 10.17795/ nmsjournal28332.

21. Wallace CJ, Robins J, Alvord LS, Walker JM. The effect of earplugs on sleep measures during exposure to simulated intensive care unit noise. Am J Crit Care 1999;8(4):210-219. DOI: 10.4037/ajcc1999. 8.4.210.

22. Hu RF, Jiang $X Y$, Zeng $Y M$, Chen $X Y$, Zhang $Y H$. Effects of earplugs and eye masks on nocturnal sleep, melatonin and cortisol in a simulated intensive care unit environment. Crit Care 2010;14(2):R66. DOI: 10.1186/cc8965.

23. Taylor KS, Murai H, Millar PJ, Haruki N, Kimmerly DS, Morris BL, et al. Arousal from sleep and sympathetic excitation during wakefulness. Hypertension 2016;68(6):1467-1474. DOI: 10.1161/ HYPERTENSIONAHA.116.08212.

24. Suzuki M, Shimamoto K, Sekiguchi $H$, Harada T, Satoya N, Inoue $Y$, et al. Arousal index as a marker of carotid artery atherosclerosis in patients with obstructive sleep apnea syndrome. Sleep Breath 2019;23(1):87-94. DOI: 10.1007/s11325-018-1664-0.

25. Zinchuk AV, Jeon S, Koo BB, Yan X, Bravata DM, Qin L, et al. Polysomnographic phenotypes and their cardiovascular implications in obstructive sleep apnoea. Thorax 2018;73(5):472-480. DOI: 10.1136/ thoraxjnl-2017-210431.

26. Winkelman C, Higgins PA, Chen YJ, Levine AD. Cytokines in chronically critically ill patients after activity and rest. Biol Res Nurs 2007;8(4): 261-271. DOI: 10.1177/1099800406298168.

27. Winkelman C. Investigating activity in hospitalized patients with chronic obstructive pulmonary disease: a pilot study. Heart Lung 2010;39(4):319-330. DOI: 10.1016/j.hrtlng.2009.09.004.

28. Morris PE, Goad A, Thompson C, Taylor K, Harry B, Passmore L, et al. Early intensive care unit mobility therapy in the treatment of acute respiratory failure. Crit Care Med 2008;36(8):2238-2243. DOI: 10.1097/ CCM.0b013e318180b90e.

29. Routsi C, Gerovasili V, Vasileiadis I, Karatzanos E, Pitsolis T, Tripodaki $\mathrm{E}$, et al. Electrical muscle stimulation prevents critical illness polyneuromyopathy: a randomized parallel intervention trial. Crit Care 2010;14(2):R74. DOI: 10.1186/cc8987.

30. Patel BK, Pohlman AS, Hall JB, Kress JP. Impact of early mobilization on glycemic control and ICU-acquired weakness in critically ill patients who are mechanically ventilated. Chest 2014;146(3):583-589. DOI: 10.1378/chest.13-2046.

31. Schweickert WD, Pohlman MC, Pohlman AS, Nigos C, Pawlik AJ, Esbrook CL, et al. Early physical and occupational therapy in mechanically ventilated, critically ill patients: a randomised controlled trial. Lancet 2009;373(9678):1874-1882. DOI: 10.1016/ S0140-6736(09)60658-9.

32. Ancoli-Israel S, Cole R, Alessi C, Chambers M, Moorcroft W, Pollak CP. The role of actigraphy in the study of sleep and circadian rhythms. Sleep 2003;26(3):342-392. DOI: 10.1093/sleep/26.3.342.

33. Kamdar BB, Kadden DJ, Vangala S, Elashoff DA, Ong MK, Martin JL, et al. Feasibility of continuous actigraphy in patients in a medical intensive care unit. Am J Crit Care 2017;26(4):329-335. DOI: 10.4037/ ajcc2017660.

34. Schwab KE, Ronish B, Needham DM, To AQ, Martin JL, Kamdar BB Actigraphy to evaluate sleep in the intensive care unit. A systematic review. Ann Am Thorac Soc 2018;15(9):1075-1082. DOI: 10.1513/ AnnalsATS.201801-0040C.

35. Knauert MP, Yaggi HK, Redeker NS, Murphy TE, Araujo KL, Pisani MA. Feasibility study of unattended polysomnography in medical intensive care unit patients. Heart Lung 2014;43(5):445-452. DOI: 10.1016/j.hrtlng.2014.06.049. 